\title{
Editorial
}

\section{Sensors Technologies and Methods for Perception Systems in Intelligent Vehicles}

\author{
Yassine Ruichek, ${ }^{1}$ Fadi Dornaika, ${ }^{2,3}$ and Maan El Badaoui El Najjar ${ }^{4}$ \\ ${ }^{1}$ University of Technology of Belfort-Montbéliard, 90010 Belfort, France \\ ${ }^{2}$ University of the Basque Country, 20018 San Sebastian, Spain \\ ${ }^{3}$ IKERBASQUE, Basque Foundation for Science, 48013 Bilbao, Spain \\ ${ }^{4}$ University of Lille, 59650 Villeneuve d'Ascq, France \\ Correspondence should be addressed to Yassine Ruichek; yassine.ruichek@utbm.fr
}

Received 8 June 2016; Accepted 8 June 2016

Copyright (C) 2016 Yassine Ruichek et al. This is an open access article distributed under the Creative Commons Attribution License, which permits unrestricted use, distribution, and reproduction in any medium, provided the original work is properly cited.

During the past decades, intelligent vehicles (advanced driving assistance systems and autonomous driving) and mobile robotic systems have received more and more attentions and developments from both research society and industry community. One of the necessary components to develop ADAS systems, driverless cars, or autonomous navigation mobile robots is perception of the surrounding vehicle environment. In intelligent vehicles, perception systems are able to sense and interpret surrounding environment based on various kinds of sensors, such as radar, sonar sensors, 2D/3D lidar, monocular/binocular/omnidirectional vision system, and inertial sensors. The perception systems provide and process sensed information for representing dynamically the content of the surrounding environment (detection, tracking, and recognition of static and dynamic objects). Therefore, processing and interpreting data provided by these sensors are required for making decision in applications related to driver assistance and vehicle control in complex environments. This special issue aims at exhibiting the latest research achievements, related to the following topics:

(i) sensors calibration methods;

(ii) sensor fusion and information integration;

(iii) sensor-based vehicle localization in GPS denied environment;

(iv) sensor-based localization and SLAM;

(v) sensor-based object detection and tracking;

(vi) sensor-based scene analysis and understanding.
This special issue received 26 papers. After reviewing process, 9 papers are accepted for publication. According to their content, the accepted papers could be classified into two classes. The first one gathers papers addressing environment perception. In this class of papers, problems discussed are camera rectification in dynamic environments, SLAM for indoor robots, map building, night-time perception, vision based vehicle speed estimation, and so forth. The second class is concerned with papers dealing with vehicle localization. The papers of this class focused on multisensory based navigations systems for cars, trains, and UAV.

Yassine Ruichek Fadi Dornaika Maan El Badaoui El Najjar 


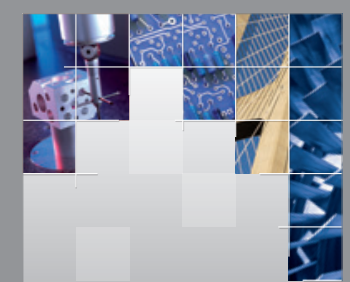

\section{Enfincering}
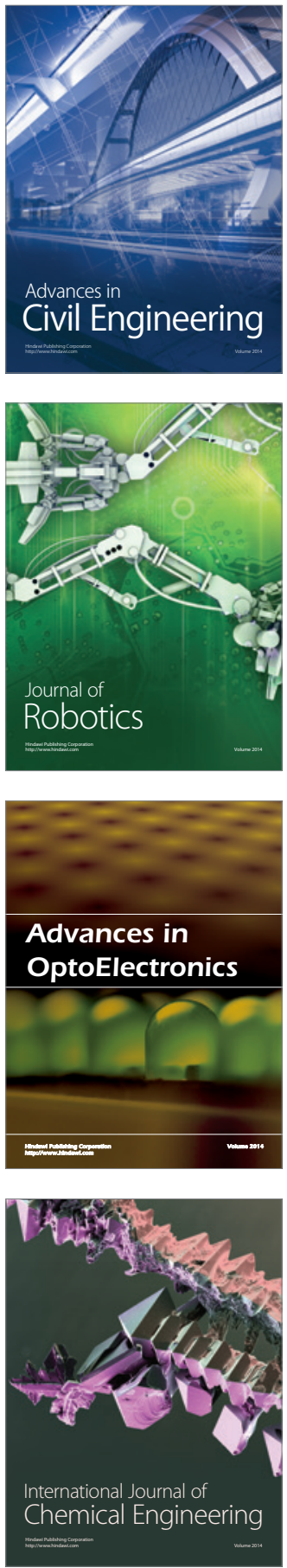

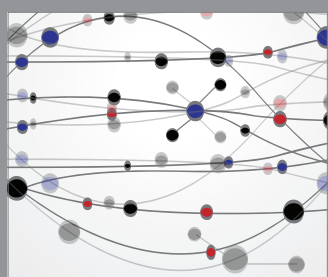

The Scientific World Journal

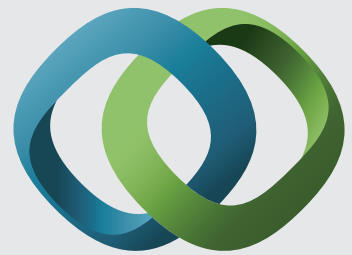

\section{Hindawi}

Submit your manuscripts at

http://www.hindawi.com
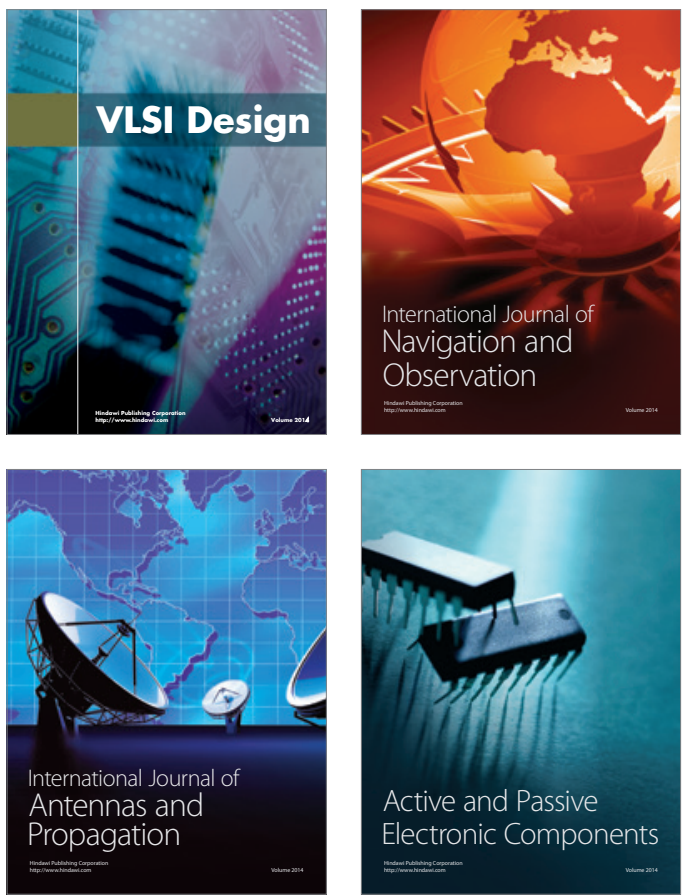
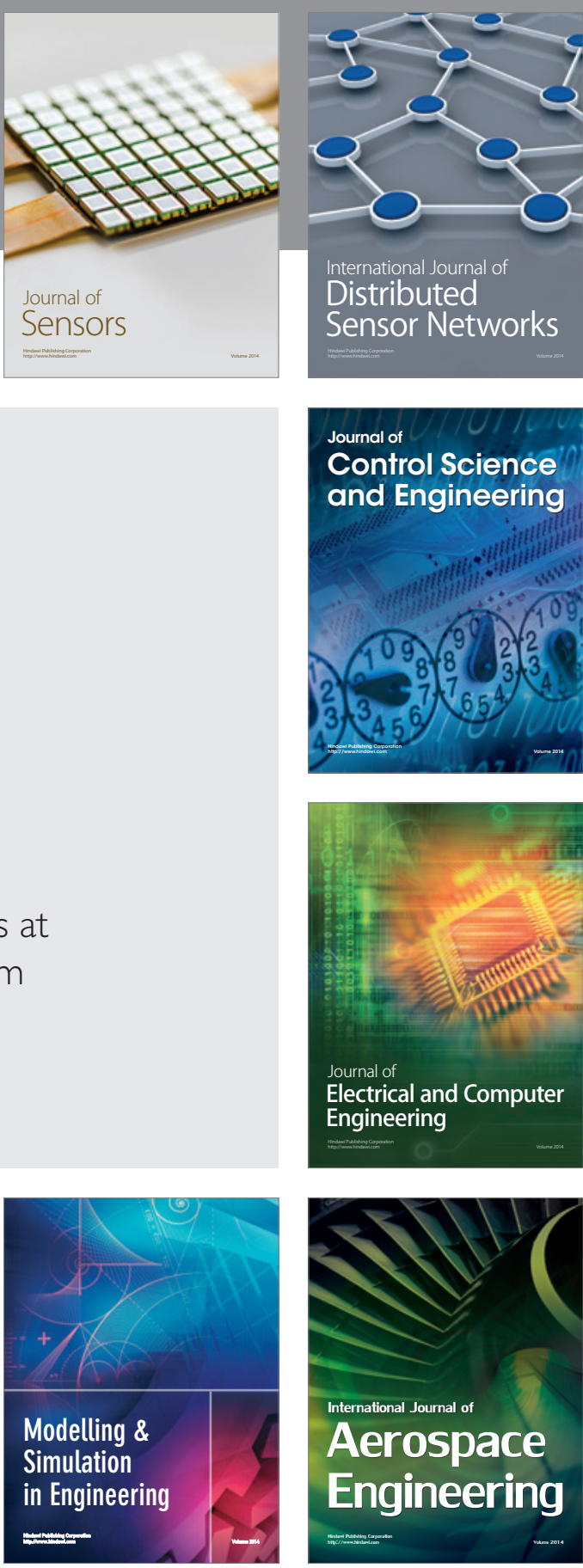

International Journal of

Distributed

Sensor Networks

Journal of

Control Science

and Engineering
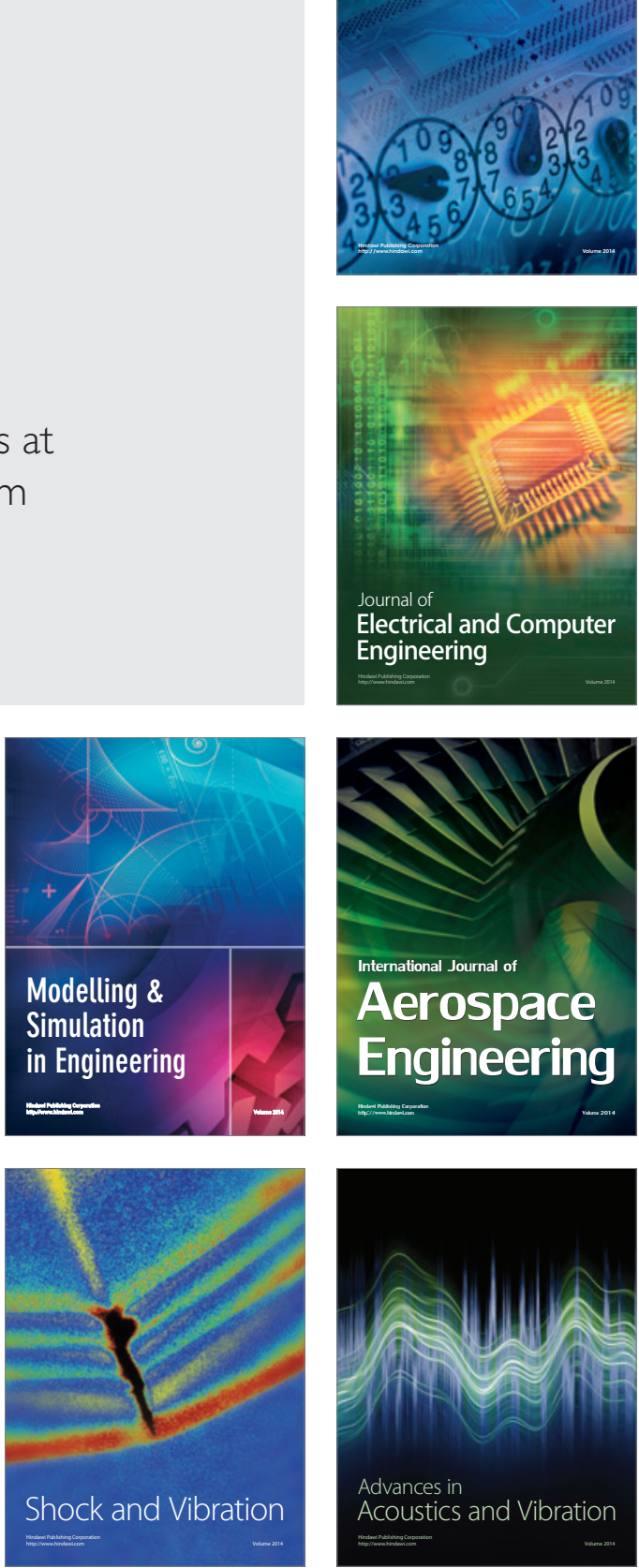